\title{
Numerical simulations in support of the design of an ultrasonic device for sub-assembly identification
}

\author{
Kevin Paumel, Tom Maurel and Christian Lhuillier
}

\begin{abstract}
In this paper, it is shown how numerical simulations can help designing an ultrasonic instrument operating in harsh conditions. To prevent fuel handling errors in sodium cooled fast reactors, the identification of fuel sub-assemblies using ultrasound is being investigated. It is based on the interpretation of a code (aligned notches) engraved on the sub-assembly head using an emitting/receiving ultrasonic sensor. This reading is performed in liquid sodium with high temperature (up to $600^{\circ} \mathrm{C}$ ) transducers. A first experiment in liquid sodium demonstrated the feasibility of this method. The reading quality and robustness depend on various parameters related to the ultrasonic beam (spectral response, focal distance, focal spot size), the code geometry (especially the notches' dimensions) and geometrical alignments. In order to avoid numerous experiments, two numerical models are developed. The first one is a finite element simulation of the sensor providing its radiated field. This model is validated with the well-known analytic solution of the Rayleigh integral; then it is applied to the sensor used in the sodium experiment. The focal distance and focal spot diameter are close to the expected values. The second simulation, using CIVA software, provides the ultrasonic scan of the code. The result is in good agreement with the sodium experiment and a first comparison with a water experiment shows that this numerical tool is relevant for easily taking into account misalignment and misorientation of the scan.
\end{abstract}

Index Terms-Focusing transducer, harsh conditions, Finite Element Method, ray-tracing code.

\section{INTRODUCTION}

In order to prevent handling errors in French sodium cooled fast reactors [1], methods identifying fuel sub-assemblies are currently considered. Liquid sodium being opaque, using ultrasound appears as a suitable solution. For decades, ultrasonic techniques have been mentionned for sub-assembly identification in liquid metal [2]-[5]. Nonetheless, as far as we know, the identication system developed for the MYRRHA reactor [6] is the only one which was described in details in the literature. This robust method uses a circular setup of twelve transducers installed on the robotic fuel manipulator, each aiming vertically at its associated notch (with varying depth) machined on the nozzle of the fuel assembly. In French sodium fast reactors, the limited available volume and dedicated sites for the transducers prompts to study in priority configurations with a single transducer aiming horizontally.

This method is based on reading, by mechanical scan, an engraved code (aligned notches) on the sub-assembly using

Kevin Paumel, Tom Maurel and Christian Lhuillier are with the CEA, DEN, Nuclear Technology Department, F-13108 Saint-Paul-lez-Durance, France, email: kevin.paumel@cea.fr an emitting/receiving ultrasonic sensor working in pulse-echo mode. This code can be compared to a bar binary code expressing the sub-assembly reference number. This reading is carried out by a sensor directly immersed in liquid sodium. It means that the sensor must withstand fuel handling conditions-about $200^{\circ} \mathrm{C}$. However, as the sensor stays in the reactor during operation, it must withstand temperature up to $580^{\circ} \mathrm{C}$. That is why a high temperature ultrasonic transducer (TUSHT® [7], [8]) is used.

In a first study [9], the feasibility of the method was demonstrated with a water experiment. This experiment also determined the resolution of the reading, i.e. the legible minimal dimensions of the notches of the code. Lately, an experiment in liquid sodium at $200^{\circ} \mathrm{C}$ [10] was carried out to demonstrate the feasibility of the method in conditions which are representative of fuel handling operations in the reactor.

In this paper, two numerical tools are studied in order to, in the future, replace numerous experiments by simulations. Indeed, the reading quality and robustness of this method depends on various parameters. Some are related to the sensor and its ultrasonic beam: spectral response, focal distance and focal spot size. Some rely on the code geometry, especially the notches' dimensions. Furthermore, the reading is sensitive to misalignment and misorientation between the ultrasonic beam and the sub-assembly head.

The first numerical tool is a model of the sensor and its beam using the finite element Method (FEM). Modeling ultrasonic transducers using the FEM has been carried out for more than two decades [11]. Unlike well-known analytic one-dimensional models [12], [13], the FEM is able to take into account the curved geometry of a focusing sensor. Even if some researchers have developed an extended KLM model [14] to efficiently compute the pressure field generated by a lens-focused axisymmetric transducer, this model neglects radial modes in the sensor, reflection angle of longitudinal waves and radial displacement at the curved interface [15]. Moreover, the FEM allows to model complex geometries of transducer. Finally, with the continuous growth of computing power, the FEM allows the calculation of the radiated field with reasonable computational cost. Besides the computation time, the FEM is criticized for its inaccuracy when simulating the propagation of acoustic waves over large distances [16]. A coupling with the Rayleigh integral [17] or using pseudospectral methods are often preferred. Nevertheless, for the short distance of interest of a focusing transducer and with a adequatly refined mesh, the accuracy may be sufficient [18], 
as it will be demonstrated in this paper.

The second model aims to assess the sensitivity of the reading method to the code geometry and its robustness to imperfect settings, such as misalignment and misorientation of the sub-assembly head. The ultrasonic scan is computed by an ultrasonic ray tracing software (CIVA [19]), using the pencil method to derive echoes with their amplitude [20], which allows computational cost much lower than the FEM.

Firstly, the reading principle of the sub-assembly identification in reactor is introduced. Then the experiment in sodium is reminded. After the parameters of the reading method are identified, and considering the heaviness of experiments, numerical simulations appear as useful tools. Thus, a FEM simulation is proposed to predict the spectral response and the radiated field characteristics of the focusing transducer. This simulation is firstly validated with the well-known analytic solution of the Rayleigh integral; then it is applied to the sensor used in the sodium experiment. Finally, a numerical simulation of the ultrasonic scan is presented. It is validated with a water experiment and then applied to the scan carried out in the sodium experiment.

\section{READING PRINCIPLE}

Several approaches for reading a code using ultrasound can be considered. This paper focuses on a robust one, i.e. that with a "favorable" reading configuration (Fig. 1). The reading consists in an upward vertical translation of a code engraved on the sub-assembly head in front of a emitter/receiver focusing ultrasonic transducer. It is performed while the sub-assembly is lifted high enough by the handling gripper so that it is completely separate from the lattice and hangs freely (no more contact). This configuration minimizes off-centering and tilt with respect to the axis of the handling machine sheath. This full separation prevents friction and jamming when moving the sub-assembly's code in front of the ultrasonic sensor. It is assumed that pendular oscillations are insignificant. Lastly, the reading is performed at the top of the sub-assembly to take advantage of less "agitated" sodium than near the core outlet.

Similarly to a bar code, the code is made of an arrangement of notches. The presence or absence of notch means respectively the 0 or 1 binary value. The cross section of the notches is triangular. In the absence of notch, when the beam hits the generatrix of the cylindrical head, the reflected signal is fully directed towards the sensor which records a significant echo. When the beam hits a notch, the oblique face of the notch deflects the echo away from the sensor which therefore records no echo.

By scanning along the vertical axis, it is possible to obtain a one-dimensional C-scan of the amplitude along the generatrix. The interpretation of the code requires post-processing this $\mathrm{C}$ scan (not discussed in this study).

A focusing sensor is used to obtain better lateral resolution than a flat sensor. The distance between the sensor's face and code must equal the sensor's focal distance so that the focal spot is located right on the code.

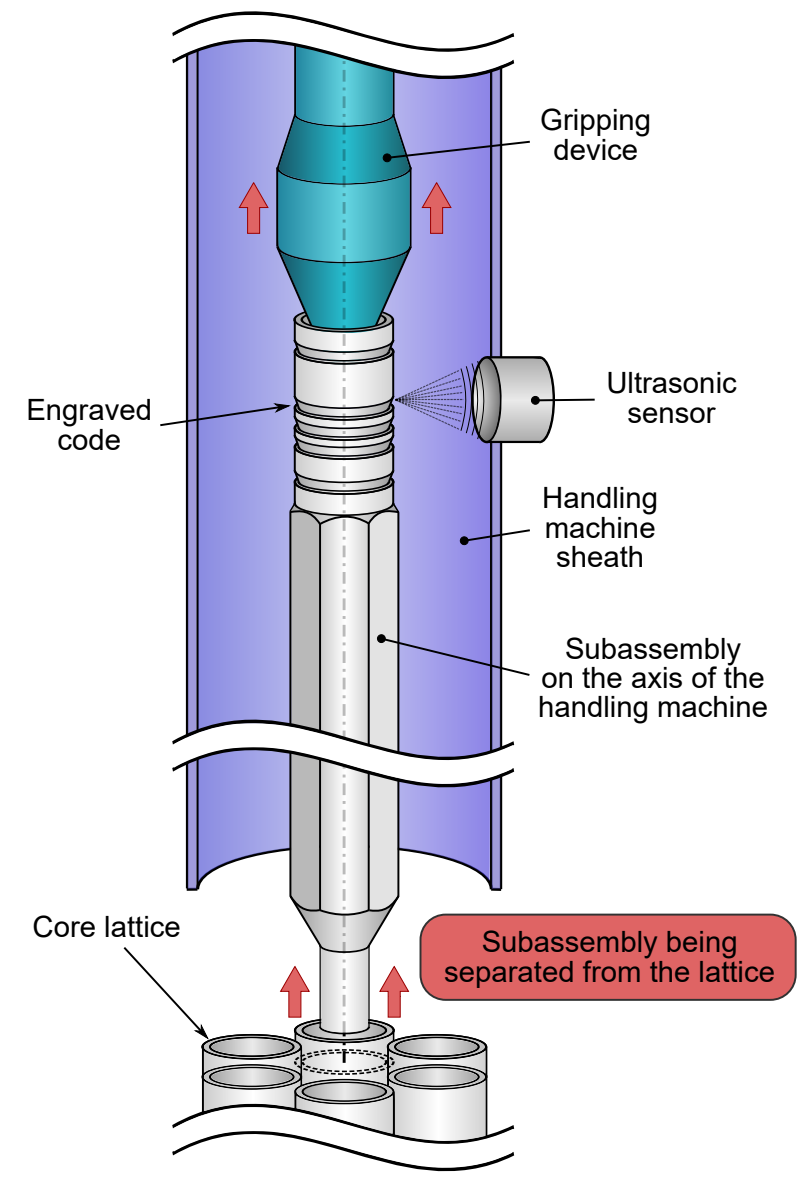

Fig. 1. Schematic diagram of a favorable reading configuration for which the sub-assembly is high enough to be completely free from the lattice.

\section{REMIND OF THE IN-SODIUM EXPERIMENT}

The experiment objective was to demonstrate the feasibility of reading a code in conditions which are representative of handling operations in the reactor, i.e. in liquid sodium at $200^{\circ} \mathrm{C}$.

\section{A. Experimental set-up}

This experiment involves scanning along the engraved code, i.e. down the generatrix of a mock-up cylindrical tube (or a cylinder arc for the mock-up in the sodium experiment) representing the sub-assembly head (Fig. 2). It is carried out under these favorable conditions:

- weak off-centering and tilt;

- straight translation of the code in relation to the ultrasonic beam;

- well-controlled displacement increment between each ultrasonic pulse emission.

The vertical movement is done by the sensor and not by the mock-up in order to simplify the process. A displacement increment of $0.5 \mathrm{~mm}$ is chosen.

This experiment was performed in a specific facility [10] in Cadarache (France) consisting of a 1,400 liters vessel of liquid sodium. Thanks to heating cables surrounding the vessel, the sodium temperature is regulated at $200^{\circ} \mathrm{C}$. 


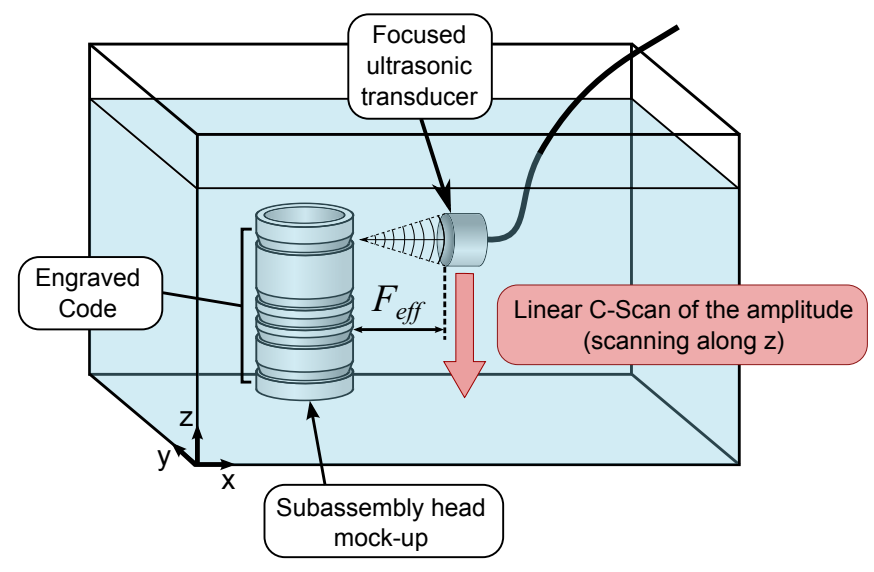

Fig. 2. Schematic diagram of the in-sodium experiment.

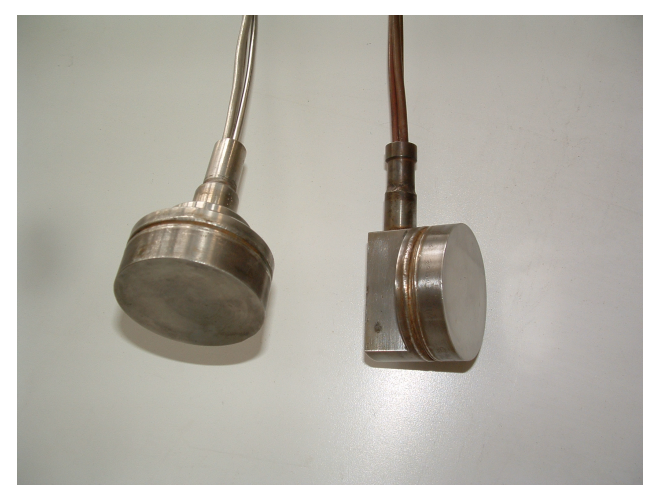

Fig. 3. High temperature ultrasonic transducer: TUSHT ${ }^{\circledR} \oslash 40 \mathrm{~mm}$ with straight (left) and $90^{\circ}$ (right) electrical wire outputs.

\section{B. Ultrasonic transducer}

1) $T U S H T^{\circledR} \oslash 40 \mathrm{~mm}:$ A high-temperature ultrasonic transducer, called in French: TUSHT ${ }^{\circledR}$ (Fig. 3), is used. This sensor is able to withstand the high temperature (up to $580^{\circ} \mathrm{C}$ ) and high radiations of the reactor core outlet under nominal power operating conditions. Other transducers withstanding only $200^{\circ} \mathrm{C}$ and offering a better damping (so a better axial resolution) could have been used for the experiment. However, as opposed to TUSHT $^{\circledR}$, those could not be permanently installed in the core outlet region.

The TUSHT $^{\circledR}$ uses high Curie temperature (about $1150^{\circ} \mathrm{C}$ ) lithium niobate $\left(\mathrm{LiNbO}_{3}\right)$ crystal as piezoelectric material. Its resistance against neutronic damage is improved thanks to a special ${ }^{7} \mathrm{Li}$ enrichment (or ${ }^{6} \mathrm{Li}$ depletion). It can withstand a $10^{21}$ fast neutron fluence and a $10^{6} \mathrm{~Gy} / \mathrm{h}$ dose rate. The crystal is bound mechanically, acoustically and electrically to the stainless steel casing and electrode by using a hard soldering technique [7], [8], [21].

The TUSHT $^{\circledR}$ is currently available in two piezoelectric crystal diameters: 15 and $40 \mathrm{~mm}$. The $40 \mathrm{~mm}$ diameter transducer was selected because it has a higher sensitivity. During the experiment, the sensor is excited with an impulse of $250 \mathrm{~V}$.

2) Focal distance: The lens of a focusing TUSHT $^{\circledR}$ is at the same time an electrode, the interface layer between the crystal and sodium and an integral part of the housing. Among such focusing TUSHT $^{\circledR}$, only a 40 mm-diameter one with a curvature radius of $R_{c}=148 \mathrm{~mm}$ was available. Its focal distance is not compatible with the confined space of the device in the vessel. Consequently, a flat transducer with a separate lens, of lower curvature radius: $R_{c}=90 \mathrm{~mm}$, is used. The interface between the planar face of the TUSHT ${ }^{\circledR}$ and the lens is a liquid sodium layer. A specific device was designed to press the lens against the sensor face to guarantee a uniform and as thin as possible sodium layer.

From the Rayleigh integral, O'Neil [17] proposed a simple theoretical expression for the acoustic pressure amplitude $p_{A}$ along the propagation axis, for sinusoidally vibrating focusing transducers with uniform normal velocity distribution on their surfaces:

$$
p_{A}(z)=\frac{\rho_{m} c_{m} u_{n} e^{-i \omega t}}{1-z / F}\left(e^{i k z}-e^{i k R}\right) .
$$

$\rho_{m}$ and $c_{m}$ are the density and sound velocity of the propagation medium, $u_{n}$ is the normal velocity amplitude on the radiator surface, $\omega$ is the angular frequency, $k=\omega / c$ is the wave number, $z$ is the distance along the propagation axis, $R=F \sqrt{1+(1-z / F)^{2}-2(1-z / F) \cos \left(\alpha_{\max }\right)}$ is the distance between the observation point and the radiator edge, where $\alpha_{\max }$ is the half-aperture angle of the radiator. In this purely theoretical configuration, $F$ corresponds to the radius of the radiator's curvature $R_{c}$. For a plane-concave lens excited on its plane side, $F$ corresponds to the geometrical focal distance and is given by the well-known formula:

$$
F=\frac{R_{c}}{1-c_{m} / c_{l}}
$$

with $c_{l}$ the longitudinal wave velocity in the lens.

More precisely, the effective focal distance $F_{\text {eff }}$ depends on the near-field limit $L_{n f}$ :

$$
L_{n f}=\frac{k a^{2}}{2 \pi}
$$

where $a$ is the radius of the excitating flat surface. Maréchal et al. [15] give an approximate relation for $F / L_{n f}<2$ :

$$
F_{\text {eff }}=\frac{F}{1+\frac{2}{3}\left(\frac{F}{L_{n f}}\right)^{4 / 3}} .
$$

To choose the best frequency range for the reading, a compromise between a high resolution (corresponding to a high frequency) and high sensitivity is necessary. As shown by the Fourier transform of the TUSHT pulse-echo response (Fig. 4), the $4.5 \mathrm{MHz}$ frequency appears to be a good choice. This also applies to the sensor used for the experiment in water (section V). Hence the distance between the sensor and mock-up generatrix is set to $F_{\text {eff }}=147 \mathrm{~mm}$.

3) Reading resolution: The reading resolution is given by the focal spot diameter of the ultrasonic beam. Assuming a non-attenuating fluid, the lateral profile in the geometric focal plane can be approximated by a Jinc function [17], [22], [23]:

$$
\operatorname{Jinc}\left(\frac{k a x}{\sqrt{F^{2}+x^{2}}}\right)=\frac{2 J_{1}\left(\frac{k a x}{\sqrt{F^{2}+x^{2}}}\right)}{\frac{k a x}{\sqrt{F^{2}+x^{2}}}}
$$




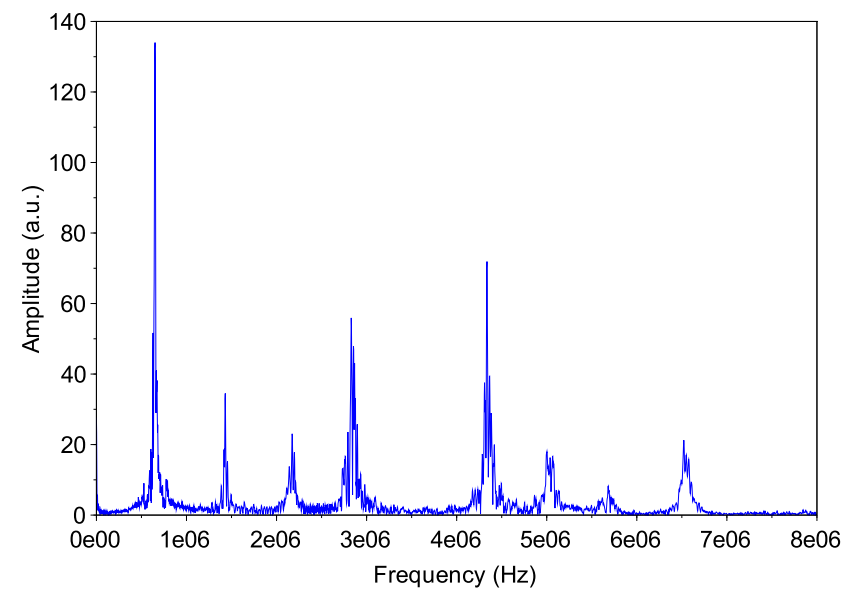

Fig. 4. Fourier transform of the echo reflected by the mock-up (in absence of notch).

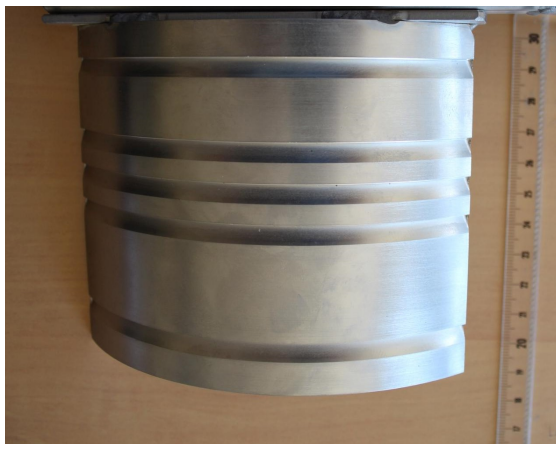

(a) Photograph of the mock-up on its supporting (b) Mock-up definition arm

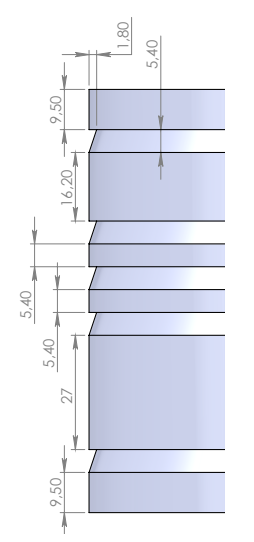

drawing
Fig. 5. Sodium experiment mock-up.

with $x$ the lateral coordinate (distance from the axis) of the beam. The focal spot diameter can be assimilated to the beam first lobe width. The coordinate $x=\sigma$ which represents the half-width of the first lobe, is given by the first zero of Bessel function $J_{1}$, which is: 3.8317 . The diameter $d$ of the focal spot is defined as follows:

$$
d=2 \sigma=2 F \sqrt{\frac{1}{\left(\frac{k a}{3.8317}\right)^{2}-1}}
$$

Furthermore, using the approximation $\sin \theta \approx \tan \theta$, the widely used formula for the first lobe radius [17] is found:

$$
\sigma=\frac{3.83 F}{k a} \text {. }
$$

Using (2) in (6) gives a focal spot diameter of $5.4 \mathrm{~mm}$.

\section{Mock-up sub-assembly head}

The mock-up of the sub-assembly head (Fig. 5) only represents the cylindrical part at the top of the sub-assembly (only a cylinder arc in this sodium experiment due to limited volume). The notch's cross-section is a right triangle present-

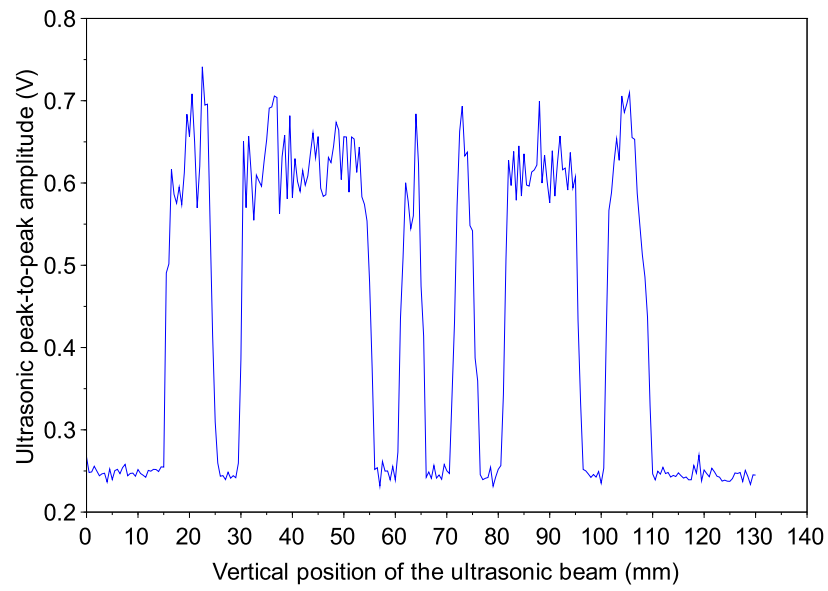

Fig. 6. One-dimensional C-scan of the sodium experiment.

ing an oblique surface to the ultrasonic beam. The notch is characterized by its width $l$ and its depth $p$.

This code only has eight notch spots, not all occupied by a notch. All notches have the same width and the notch spots are equally spaced by a distance equal to the notch width. The code is 01100010 . To ensure a good separation between the two binary states with respect to specular reflection of the signal received by the ultrasonic sensor, while limiting the code height, the notch's width $l$ is set to the focal spot diameter $d=2 \sigma=5.4 \mathrm{~mm}$.

The height of the code is the sum of all notch spots and all spacings between them. The number of bits required for the identification is expected to be greater, especially if additional criteria of redundancy or error detection are considered.

\section{Result}

The output data is a one-dimensional amplitude C-scan for a full vertical sweep along the mock-up (Fig. 6). More precisely, each point of this $\mathrm{C}$-Scan corresponds to the peak-to-peak amplitude of the filtered A-Scan at a given position of the transducer. The filtering consists in a high-pass Butterworth's filtering (cut-off frequency at $4.5 \mathrm{MHz}$ ) in order to remove low frequencies which lead to a loss of resolution.

The code appears clearly on the graph. However, while the 0-bit (presence of a notch) is well delimited in amplitude, the 1-bit is subjected to noise. This is due to the interface between the sensor and the lens which induces an acoustic impedance discontinuity and so a weak signal-to-noise ratio. A much cleaner result is expected with a real focusing TUSHT ${ }^{\circledR}$ (i.e. with a lens directly incorporated to the sensor).

With a relevant choice of amplitude thresholds, a relatively simple algorithm could convert this C-Scan into a binary code expressing the sub-assembly type and number. Hence, the reading quality depends on the contrast between amplitude of "notch" and amplitude "of no-notch". This contrast is strongly affected by the misalignment and the misorientation between the ultrasonic beam and the sub-assembly head. 


\section{Finite ELEMENT MODELLING OF THE SENSOR AND ITS RADIATED FIELD}

In order to verify the analytic assessments derived in sections III-B2 \& III-B3 and to have a powerful tool available for designing sensors, a finite element model of the sensor and the propagation medium is implemented.

\section{A. Model set-up}

The model is based on the mechanical and electrical behaviors of the sensor's components. It computes the ultrasonic field given a harmonic electrical excitation applied at the ports of the piezoelectric cristal. It's a frequency domain studysimplifying the mathematical expression of the problem.

The model, implemented on COMSOL ${ }^{\circledR}$, uses three constitutive relations :

- The stainless steel (AISI 304L) of the two electrodes and the lens is given a visco-elastic isotropic behavior.

- The Lithium Niobate of the piezoelectric cristal is given a coupled piezoelectric and elastic behavior (which is anisotropic and depends on the crystal's orientation).

- The liquid sodium of the propagation medium is given a non-viscous fluid-acoustic behavior.

Given the symmetry of the system, a 2D axisymetric geometry is chosen to reduce the computing cost.

The mesh is made of triangular elements with quadratic interpolation functions. The elements' size is chosen so that a wavelength is discretized by at least 8 elements in any material. Some convergence tests have shown that this criterion offers a satisfying compromise between computing cost and accuracy. As a consequence, the element's size is determined by the medium in which the celerity of transverse or longitudinal waves is minimal. Here, the celerity of longitudinal waves in sodium is the lowest $\left(2472 \mathrm{~m} . \mathrm{s}^{-1}\right.$ at $\left.200^{\circ} \mathrm{C}\right)$, even lower than the transverse waves in stainless steel (greater than $3000 \mathrm{~m} . \mathrm{s}^{-1}$ ). As an example, the mesh consists of five million elements for a sodium domain $55 \mathrm{~mm}$ in diameter and about $200 \mathrm{~mm}$ long at a $4.5 \mathrm{MHz}$ frequency. The associated maximum element size is around $70 \mu \mathrm{m}$.

The interfaces between materials are given a continuous displacement boundary. Perfectly Matched Layers (PML) are used to reproduce an infinite volume by preventing reflections at the edge of the propagation domain. Every other external boundary is considered free. A voltage amplitude of $200 \mathrm{~V}$ is applied at the back of the piezoelectric crystal while the other port is grounded.

For any given frequency and any geometry of the sensorlens system, the simulation computes the associated radiated field. The focal distance and shape of the focal spot can then be derived.

\section{B. The Rayleigh's configuration}

To validate the model for wave propagation in sodium, a comparison to the analytic solutions-equations (1) and (5)has been carried out. Its principle is to compute the acoustic pressure field radiated by the curved interface of the lens for a uniform normal velocity. The FEM simulation gives the same pressure field as the analytical model (Fig. 7).

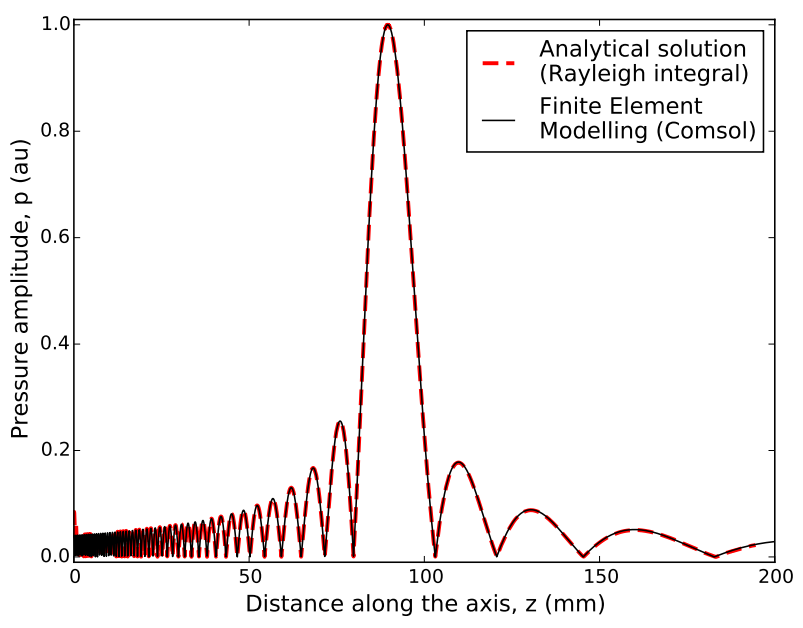

(a) Along the propagation axis $\mathrm{Z}$

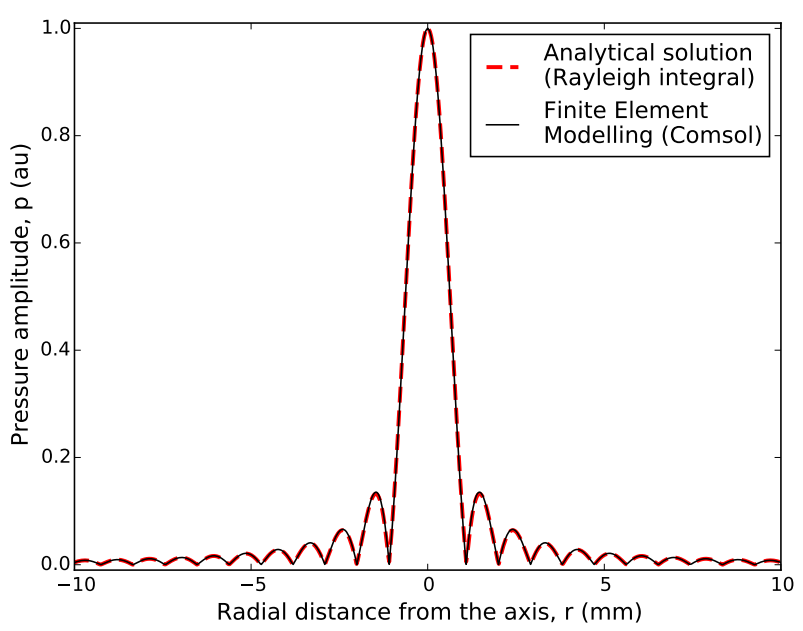

(b) Along the radial axis on the focal spot

Fig. 7. Evaluation of the pressure field's amplitude for the Rayleigh's configuration (2D field and evaluation axes on figure 9).

This validates the hypotheses of the FEM model, mesh and boundary conditions. It also confirms that the error accumulated-inherent to finite elements methods-along distances relevant for this study (typically focal length) remains negligible.

\section{The whole TUSHT ${ }^{\circledR}$ configuration}

The model is then applied to the whole TUSHT $^{\circledR}$. The resulting pressure field along the propagation axis shows that the maximum of pressure amplitude beyond the near field is about $146 \mathrm{~mm}$ from the sensor (Fig. 8). This focal distance is close to the approximate analytical value $147 \mathrm{~mm}$, given by the equation (4) in section III-B2. Besides, the width of the first lobe observed on the radial axis at this focal distance is in good agreement with the focal spot diameter of $5.4 \mathrm{~mm}$ calculated by the equation (6) in section III-B3.

Figure 9 shows a 2D view of the pressure field in both cases: the Rayleigh's configuration and the whole TUSHT ${ }^{\circledR}$. This representation offers some information on the spatial distribution of acoustic energy. 


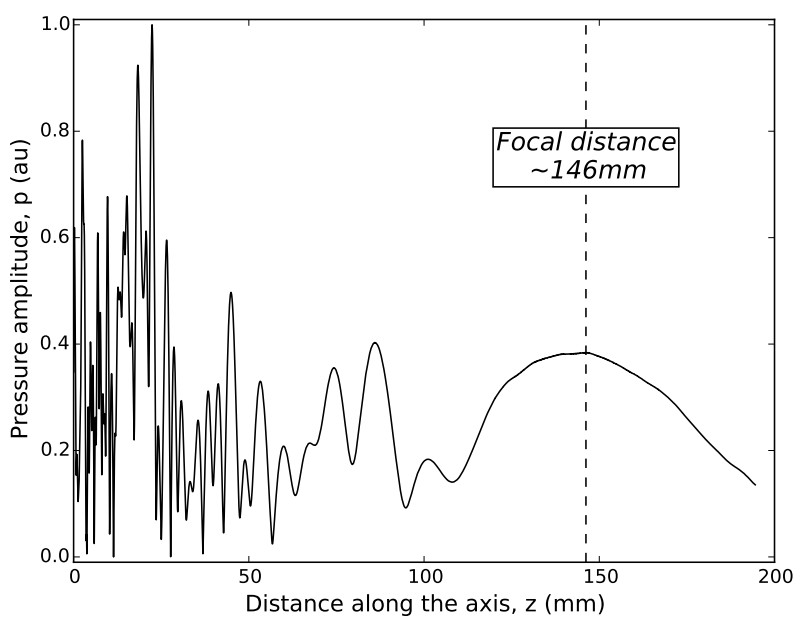

(a) Along the propagation axis $\mathrm{Z}$

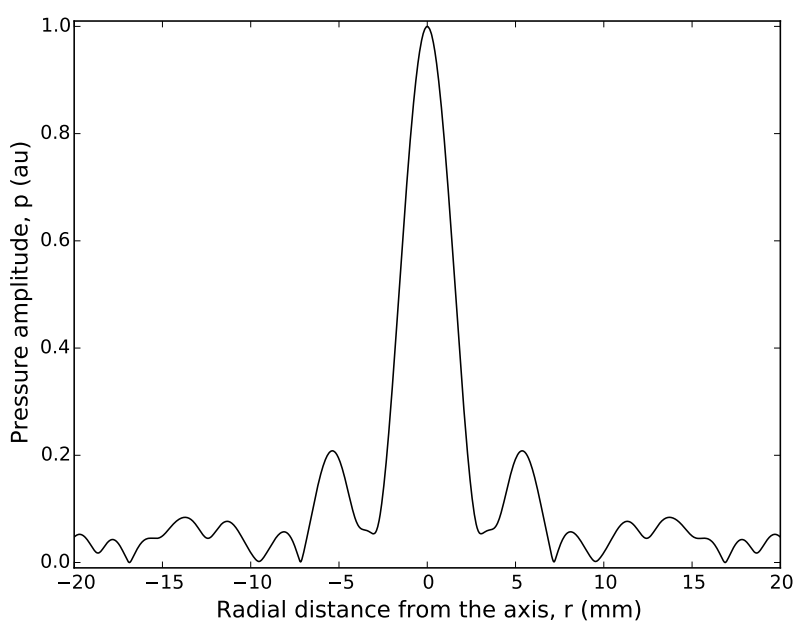

(b) Along the radial axis on the focal spot

Fig. 8. Evaluation of the pressure field's amplitude for the whole TUSHT ${ }^{\circledR}$ configuration (2D field and evaluation axes on figure 9).

\section{Conclusion on FEM model}

These results show the relevance and accuracy of the FEM simulation to evaluate the radiated field of a non-standard focusing transducer. In this first harmonical approach, the agreement between analytic formulae and FEM simulation confirms the consistency of both tools for designing the sensor. The FEM simulation will bring its benefit in particular for complex geometries of the sensor's components, for example non-axisymmetric lenses. The direct $2 \mathrm{D}$ representation already provides useful information about the energetical topology of the beam.

The next step is to upgrade the model to take into account the multifrequency feature of the excitation and to obtain a pulse response of the transducer.

\section{NUMERICAL SIMULATION OF THE SCAN WiTH CIVA}

Numerical simulation allows to modify reading parameters of the code (focal distance of the sensor, geometry and size of the code, size of the assembly head, etc.) very easily while obtaining results very quickly and at low cost. Moreover, a

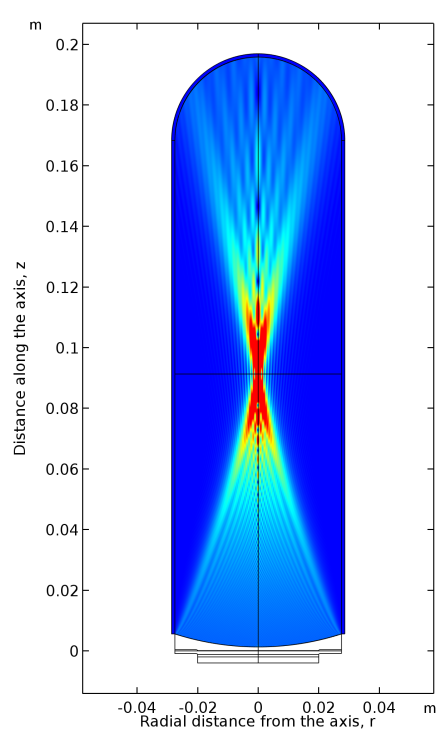

(a) Rayleigh's configuration

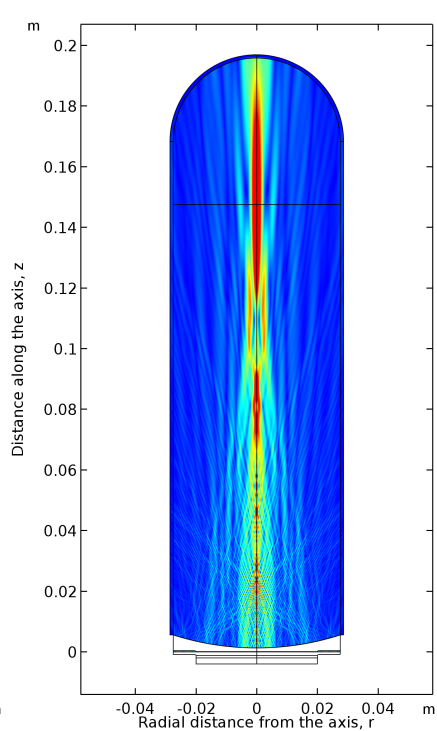

(b) Whole TUSHT ${ }^{\circledR}$ configuration
Fig. 9. 2D view of the radiated field of the sensor at $4.5 \mathrm{MHz}$ with depiction of the evaluation axis (z-axis and focal spot r-axis).

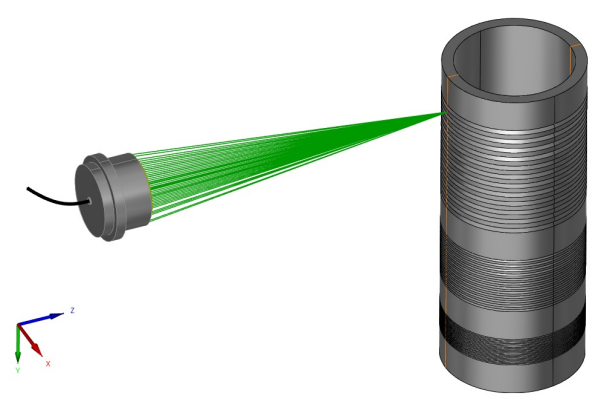

Fig. 10. 3D view of the CIVA simulation.

numerical model simplifies the implementation misalignment and misorientation to determine the maximal admissible errors of the reading method. The reading configuration is modeled with CIVA software (Fig. 10).

First, the model needs to be validated. Simulation results are compared to a previous water experiment [9] in which a real focusing TUSHT (with $R_{c}=148 \mathrm{~mm}$ ) was used. In this experiment, the mock-up consists of three codes of twelve notches with various notch geometries. All the spots are taken by a notch. The notches have the following dimensions:

- Width $l$ (same as spacing between notches) with the following values: $0.2 d=0.8 \mathrm{~mm}, 0.4 d=1.6 \mathrm{~mm}$ and $0.8 d=3.2 \mathrm{~mm}$, with $d$ given by (6) using (2). $l$ varies from one code to the next.

- Depth $p$ so that the ratio $l / p$ corresponds to the following values: 2, 3 and 4 (but its influence was found to be insignificant). $p$ varies in a same code.

An experimental signal is implemented as input data in the CIVA model. It should be noted that, for future studies, the objective is to implement a computed signal derived from the FEM model to avoid the need of experimental recording.

Figure 11 shows the one-dimensional C-Scan. A good agreement is observed between experimental data and numeri- 


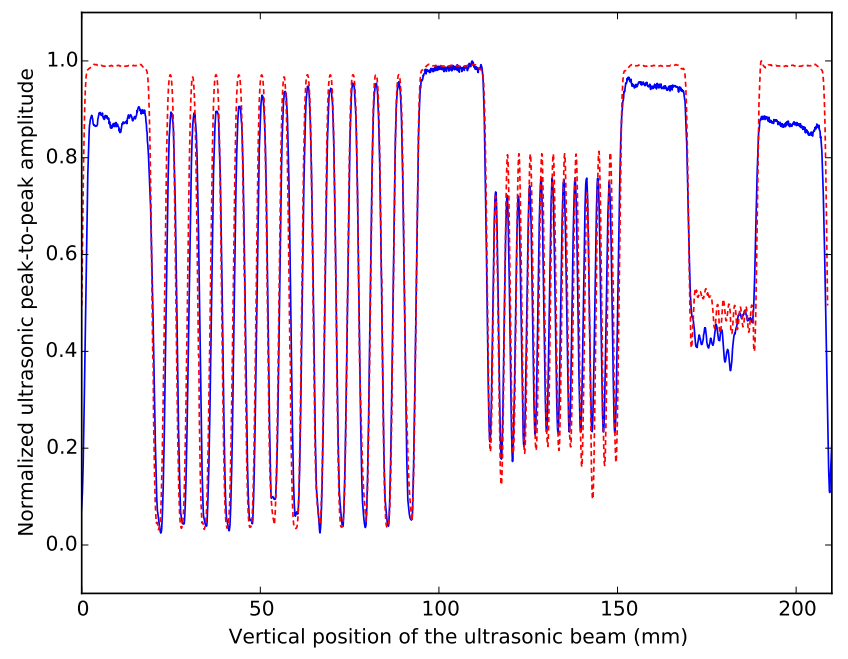

Fig. 11. One-dimensional C-scan of the amplitude of the water experiment. Red dotted: CIVA simulation, blue: experiment.

cal data from CIVA simulation with full incident beam setting. This result is better than the former one [10] obtained with the plane wave approximation setting. The noise of the signal observed in the sodium experiment has been greatly reduced by using a real focusing TUSHT $^{\circledR}$ in the water experiment. Moreover, the reading exhibits a very good quality, for the largest notch width at least, although the width is smaller than the focal spot.

A deviation for the global envelope is observed though in the form of a slight curvature of the code. This deviation between simulation and experiment could be caused by slight misalignment and misorientation in the experiment. It is indeed very difficult to accurately set the orientation of the sensor with respect to the mock-up generatrix for every position of the sweep. In an updated computation with CIVA, a 0.7 degree rotation of the mock-up around the beam axis at middle position of the sweep is introduced. With that correction, the numerical result is even closer to the experiment (Fig. 12).

Hence, the CIVA model can reasonably be considered as validated. It is then used to simulate the sodium experiment (Fig. 13). Again, for this experiment, a satisfying agreement is obtained between the model and the experiment. The low signal to noise ratio in the experiment may explain the overestimation of the contrast-between the presence and absence of notch-by the simulation.

\section{CONCLUSION}

This study showed the relevance of numerical simulation in support of the design process of an ultrasonic instrument. It allows to save experiments in harsh conditions.

A method for sub-assembly identification in liquid sodium using ultrasound has been proposed. It is based on the reading of a code engraved on the sub-assembly head by an emitting/receiving ultrasonic sensor. This code, whose height doesn't exceed two hundreds millimeters, comprises aligned notches (of a few millimeters of width) deflecting the ultrasonic beam. This reading is performed in sodium with a

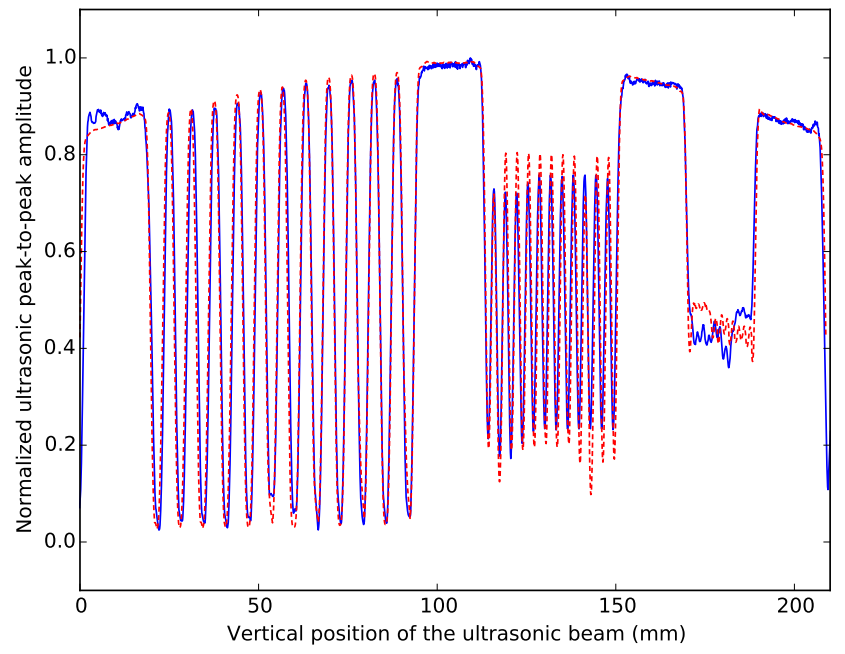

Fig. 12. One-dimensional C-scan of the amplitude of the water experiment. Red dotted: CIVA simulation with 0.7 degree rotation of the mock-up around the beam axis at middle position of the sweep, blue: experiment.

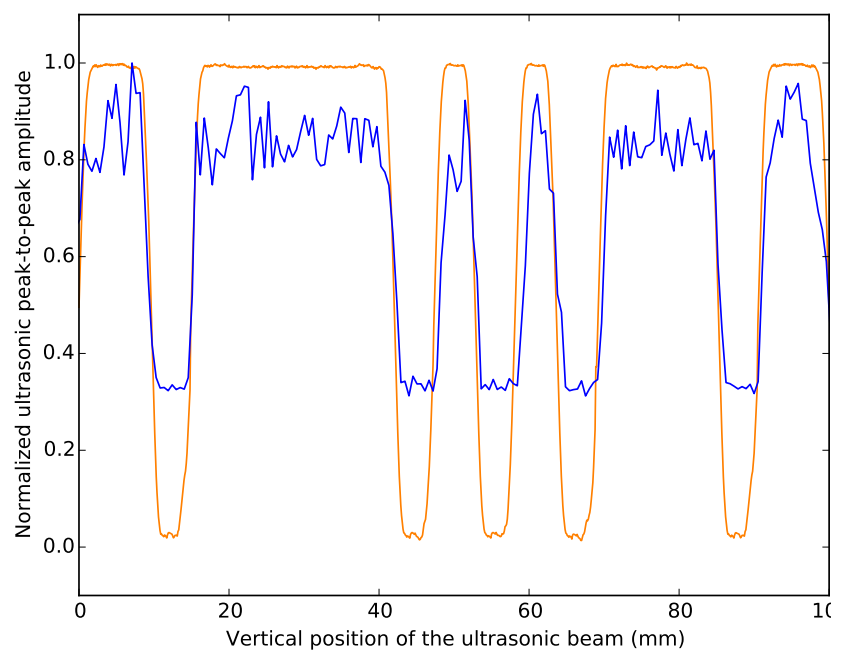

Fig. 13. One-dimensional C-scan of the amplitude of the sodium experiment. Orange: CIVA simulation, blue: experiment.

high temperature transducer, the TUSHT ${ }^{\circledR}$. The resulting scan can be compared to a binary code expressing the sub-assembly reference number.

A representative experiment in liquid sodium at $200^{\circ} \mathrm{C}$ demonstrated the feasibility of this method for a notch width equaling the focal spot diameter of the focusing sensor.

In order to avoid numerous experiments, two numerical tools have been studied. The first is a finite element model of the sensor and its radiated field. Concerning wave propagation in sodium, the model has been validated by comparison with well known analytic expressions. The model has then been applied to the TUSHT $^{\circledR}$. The focal distance and spot diameter obtained are in agreement with the analytic formulae. The second numerical simulation uses CIVA software. It provides the ultrasonic scan of the code. An in-water experiment validated the model and proved its efficiency to take into account misorientation of the ultrasonic beam with respect to the code (misalignment may be treated as well). Concerning 
the in-sodium experiment, the simulation also gives results with a satisfying agreement despite the low signal to noise ratio.

In a near future, the FEM model might be upgraded to take into account the multi-frequency feature of the excitation. The resulting computed signal could then be used as an input data in the CIVA simulation.

\section{REFERENCES}

[1] F. Dechelette, F. Morin, G. Laffont, G. Rodriguez, E. Sanseigne, S. Christin, X. Mognot, and A. Morcillo, "Study and Evaluation of Innovative Fuel Handling Systems for Sodium-Cooled Fast Reactors: Fuel Handling Route Optimization," Science and Technology of Nuclear Installations, 2014.

[2] J. Spanner, "Preliminary development of in-service inspection methods for LMFBRs," in Specialists Meeting on "Inservice Inspection and Monitoring of LMFBR's", Bensberg, Germany, Mar. 1976.

[3] C. C. Scott, P. R. Huebotter, and R. C. Callen, "Potential applications for an under-sodium ultrasonic scanning device," Tech. Rep. Atomic Power Development Associates Report APDA-205, Jul. 1967.

[4] R. N. Ord and R. W. Smith, "Development of Under- Sodium Ultrasonic Scanner for in- Reactor Surveillance," Hanford Engineering Development Laboratory, Richland, Washington, Tech. Rep. Technical Report: HEDL-TME 72-91, 1972.

[5] C. K. Day and R. W. Smith, "Under-Sodium Viewing," in IEEE Ultrasonics Symposium, Monterey, California, USA, Nov. 1973, pp. 191-194.

[6] D. V. Dyck and M. Dierckx, "An Ultrasonic Fuel Identification System for Liquid Metal Cooled Reactors Resilient Against Multiple Transducer Failures," IEEE Transactions on Nuclear Science, vol. 61, no. 4, pp. 2291-2299, Aug. 2014.

[7] C. Lhuillier, B. Marchand, J. M. Augem, J. Sibilo, and J. F. Saillant, "Generation IV nuclear reactors: Under sodium ultrasonic transducers for Inspection and Surveillance," in 2013 3rd International Conference on Advancements in Nuclear Instrumentation, Measurement Methods and their Applications (ANIMMA), Jun. 2013, pp. 1-6.

[8] C. Lhuillier, "Method for Manufacturing a High-Temperature Ultrasonic Transducer Using a Lithium Niobate Crystal Brazed with Gold and Indium," France Patent US20 140215784 A1, Aug., 2014.

[9] K. Paumel and C. Lhuillier, "Identifying subassemblies by ultrasound to prevent fuel handling error in sodium fast reactors: First test performed in water," in 2015 4th International Conference on Advancements in Nuclear Instrumentation Measurement Methods and their Applications (ANIMMA), Apr. 2015, pp. 1-7.

[10] K. Paumel, "Subassembly identification by ultrasound in sodium cooled fast reactors," in 2018 IEEE International Instrumentation and Measurement Technology Conference (I2MTC), May 2018, pp. 1-6.

[11] N. N. Abboud, G. L. Wojcik, D. K. Vaughan, J. Mould, D. J. Powell, and L. Nikodym, "Finite element modeling for ultrasonic transducers," in Ultrasonic Transducer Engineering: Medical Imaging 1998, K. K. Shung, Ed. Bellingham: Spie-Int Soc Optical Engineering, 1998, vol. 3341, pp. 19-42, wOS:000074538600002.

[12] M. Redwood, "Transient Performance of a Piezoelectric Transducer," The Journal of the Acoustical Society of America, vol. 33, no. 4, pp. 527-536, Apr. 1961. [Online]. Available: https://asa.scitation.org/doi/10.1121/1.1908709

[13] R. Krimholtz, D. A. Leedom, and G. L. Matthaei, "New equivalent circuits for elementary piezoelectric transducers," Electronics Letters, vol. 6 , no. 13, pp. 398-399, Jun. 1970.

[14] P. Maréchal, F. Levassort, L.-P. Tran-Huu-Hue, and M. Lethiecq, "Lens-focused transducer modeling using an extended KLM model," Ultrasonics, vol. 46, no. 2, pp. 155-167, May 2007. [Online]. Available: http://www.sciencedirect.com/science/article/pii/S0041624X07000194

[15] _ _Effect of Radial Displacement of Lens on Response of Focused Ultrasonic Transducer," Japanese Journal of Applied Physics, vol. 46, no. 5R, p. 3077, May 2007.

[16] E. Filoux, S. Calle, R. Lou-Moeller, M. Lethiecq, and F. Levassort, "3-D numerical modeling for axisymmetrical piezoelectric structures: application to high-frequency ultrasonic transducers," IEEE Transactions on Ultrasonics, Ferroelectrics, and Frequency Control, vol. 57, no. 5, pp. 1188-1199, May 2010.

[17] H. T. O'Neil, "Theory of Focusing Radiators," The Journal of the Acoustical Society of America, vol. 21, no. 5, pp. 516-526, Sep. 1949.
[18] P. N. Bilgunde and L. J. Bond, "A 2d finite element simulation of liquid coupled ultrasonic NDT system," AIP Conference Proceedings, vol. 1650, no. 1, pp. 1543-1552, Mar. 2015. [Online]. Available: https://aip.scitation.org/doi/abs/10.1063/1.4914773

[19] S. Mahaut, S. Chatillon, M. Darmon, N. Leymarie, R. Raillon, and P. Calmon, "An Overview of Ultrasonic Beam Propagation and Flaw Scattering Models in the Civa Software," in Review of Progress in Quantitative Nondestructive Evaluation, Vols $29 a$ and 29b, D. O. Thompson and D. E. Chimenti, Eds., vol. 1211. Melville: Amer Inst Physics, 2010, pp. 2133-2140.

[20] N. Gengembre and A. Lhémery, "Pencil method in elastodynamics: application to ultrasonic field computation," Ultrasonics, vol. 38, no. 1, pp. 495-499, Mar. 2000.

[21] M. Giot, L. Vermeeren, A. Lyoussi, C. Reynard-Carette, C. Lhuillier, P. Mégret, F. Deconinck, and B. S. Gonçalves, "Nuclear instrumentation and measurement: a review based on the ANIMMA conferences," EPJ Nuclear Sciences \& Technologies, vol. 3, p. 33, 2017.

[22] B. G. Lucas and T. G. Muir, "The field of a focusing source," The Journal of the Acoustical Society of America, vol. 72, no. 4, pp. 1289 1296, Oct. 1982.

[23] A. Goldstein, "Steady state spherically focused, circular aperture beam patterns," Ultrasound in Medicine \& Biology, vol. 32, no. 10, pp. 14411458 , Oct. 2006 